\title{
A Formula for the Numerical Range of Elementary Operators
}

\author{
M. Barraa \\ Department of Mathematics, Faculty of Sciences Semlalia, Marrakech, Morocco \\ Correspondence should be addressed to M. Barraa; barraa@hotmail.com
}

Received 10 November 2013; Accepted 9 February 2014; Published 20 March 2014

Academic Editors: M. Lindstrom and C. Zhu

Copyright (C) 2014 M. Barraa. This is an open access article distributed under the Creative Commons Attribution License, which permits unrestricted use, distribution, and reproduction in any medium, provided the original work is properly cited.

Let $B(H)$ be the algebra of bounded linear operators on a complex Hilbert space $H$. For $k$-tuples of elements of $B(H)$, $A=$ $\left(A_{1}, \ldots, A_{k}\right)$ and $B=\left(B_{1}, \ldots, B_{k}\right)$, let $R_{A, B}$ denote the elementary operator on $B(H)$ defined by $R_{A, B}(X)=\sum_{i=1}^{k} A_{i} X B_{i}$. In this paper, we prove the following formula for the numerical range of $R_{A, B}: V\left(R_{A, B}, B(B(H))\right)=\left[\cup_{U \in U(H)} W\left(\sum_{i=1}^{k} U A_{i} U^{*} B_{i}\right)^{-}\right]^{-}$, where $U(H)$ is the set of unitary operators.

\section{Introduction}

Let $\mathscr{A}$ be a complex Banach algebra with unit. For $k$-tuples of elements of $\mathscr{A}, a=\left(a_{1}, \ldots, a_{k}\right)$ and $b=\left(b_{1}, \ldots, b_{k}\right)$, let $R_{a, b}$ denote the elementary operator on $\mathscr{A}$ defined by

$$
R_{a, b}(x)=\sum_{i=1}^{k} a_{i} x b_{i}
$$

This is a bounded linear operator on $\mathscr{A}$. Some interesting cases are the generalized derivation $\delta_{a, b}(x)=a x-x b$ and the multiplication $M_{a, b}(x)=a x b$ for $a, b, x \in A$.

The numerical range of $a \in \mathscr{A}$ is defined by

$$
V(a, \mathscr{A})=\{f(a): f \in S\},
$$

where $S$ is the set of normalized states in $\mathscr{A}$ :

$$
S=\left\{f \in A^{*},\|f\|=1=f(e)\right\} .
$$

See [1-3]. It is well known that $V(a, \mathscr{A})$ is convex and closed and contains the spectrum $\sigma(a)$. For $\mathscr{A}=B(X)$, the algebra of bounded linear operators on a normed space $X$, and $T \in B(X)$, in addition to $V(T, B(X))$, we have the spatial numerical range of $T$, given by

$$
\begin{aligned}
& W(T) \\
& =\left\{f(T x): x \in X,\|x\|=1, f \in X^{*},\|f\|=f(x)=1\right\},
\end{aligned}
$$

and it is known that $V(T, B(X))=\overline{\operatorname{co}} W(T)$, the closed convex hull of $W(T)$. In the case of a Hilbert space $X=H$, then

$$
W(T)=\{\langle T x, x\rangle: x \in H,\|x\|=1\}
$$

is convex but not closed in general and $V(T, B(H))=W(T)^{-}$.

Many facts about the relation between the spectrum of $R_{a, b}$ and the spectrums of the coefficients $a_{i}$ and $b_{i}$ are known. This is not the case with the relation between the numerical range of $R_{a, b}$ and the numerical ranges of $a_{i}$ and $b_{i}$. Apparently, the only elementary operator on a Hilbert space for which the numerical range is computed is the generalized derivations [4-8]. It is Fong [4] who first gives the following formula:

$$
W\left(\delta_{T}\right)=W(T)^{-}-W(T)^{-},
$$

where $\delta_{T}$ is the inner derivation defined by $\delta_{T}(X)=T X-$ $X T$. Shaw [7] (see also $[5,6]$ ) extended this formula to generalized derivations in Banach spaces. For a good survey of the numerical range of elementary operators, you can see [9], where the following problem is posed.

Problem. Determine the numerical range of the elementary operator $R_{A, B}$.

In this paper, we give a formula that answers this problem. 


\section{Main Result}

The following theorem is the main result in this paper.

Theorem 1. Let $H$ be a complex Hilbert space. Let $A=$ $\left(A_{1}, \ldots, A_{k}\right)$ and $B=\left(B_{1}, \ldots, B_{k}\right)$ be two $k$-tuples of elements in $B(H)$. Then, one has

$$
V\left(R_{A, B}, B(B(H))\right)=\left[\bigcup_{U \in U(H)} W\left(\sum_{i=1}^{k} U A_{i} U^{*} B_{i}\right)^{-}\right]^{-}
$$

In particular for multiplication and generalized derivation, one has $(A, B \in B(H))$ :

$$
\begin{gathered}
V\left(M_{A, B}, B(B(H))\right)=\left[\bigcup_{U \in U(H)} W\left(A U B U^{*}\right)^{-}\right]^{-}, \\
V\left(\delta_{A, B}, B(B(H))\right)=\left[\bigcup_{U \in U(H)} W\left(U^{*} A U-B\right)^{-}\right]^{-} .
\end{gathered}
$$

From Fong's formula (see $[4,6,10]$ ), we can deduce the following.

Corollary 2. For $A, B \in B(H)$, one has

$$
\begin{aligned}
W(A)^{-}-W(B)^{-} & =\left[\bigcup_{U \in U(H)} W\left(U A U^{*}-B\right)^{-}\right]^{-} \\
& =\left[\bigcup_{U \in U(H)} W\left(A-U B U^{*}\right)^{-}\right]^{-} .
\end{aligned}
$$

\section{Proof of the Main Result}

One of the keys to the proof of our main result is the following lemma.

Lemma 3. Let $A=\left(A_{1}, \ldots, A_{k}\right)$ and $B=\left(B_{1}, \ldots, B_{k}\right)$ be two $k$-tuples of elements in $B(H)$. Then, one has

$$
W\left(\sum_{i=1}^{k} A_{i} B_{i}\right) \subset V\left(R_{A, B}, B(B(H))\right) .
$$

In particular, for $A, B \in B(H)$, one has

$$
\begin{gathered}
W(A B) \subset V\left(M_{A, B}, B(B(H))\right), \\
W(A-B) \subset V\left(\delta_{A, B}, B(B(H))\right) .
\end{gathered}
$$

Proof. Let $\lambda \in W\left(\sum_{i=1}^{k} A_{i} B_{i}\right)$; by definition, there exists $x \in H$ with $\|x\|=1$ such that

$$
\lambda=\left\langle\sum_{i=1}^{k} A_{i} B_{i} x, x\right\rangle=\operatorname{tr}\left(\sum_{i=1}^{k} A_{i} B_{i}(x \otimes x)\right) .
$$

Here, $\operatorname{tr}(\cdot)$ is the linear form trace. Let $\phi_{x \otimes x}$ be the linear form defined by

$$
\phi_{x \otimes x}(X)=\operatorname{tr}(X(x \otimes x))=\langle X x, x\rangle .
$$

This is a bounded linear form on $B(H)$, with norm being equal to 1 , because

$$
\left\|\phi_{x \otimes x}\right\|=\|x \otimes x\|=1
$$

Since

$$
\phi_{x \otimes x}\left(I_{H}\right)=\operatorname{tr}(x \otimes x)=\langle x, x\rangle=1,
$$

the form $\phi_{x \otimes x}$ is a state on $B(H)$. So,

$$
\begin{aligned}
\phi_{x \otimes x}\left(R_{A, B}\left(I_{H}\right)\right) \in V\left(M_{A, B}, B(B(H))\right), \\
\phi_{x \otimes x}\left(R_{A, B}\left(I_{H}\right)\right)=\phi_{x \otimes x}\left(\sum_{i=1}^{k} A_{i} B_{i}\right) \\
=\operatorname{tr}\left(\sum_{i=1}^{k} A_{i} B_{i}(x \otimes x)\right)=\left\langle\sum_{i=1}^{k} A_{i} B_{i} x, x\right\rangle \\
=\lambda .
\end{aligned}
$$

Hence, $W\left(\sum_{i=1}^{k} A_{i} B_{i}\right) \subset W\left(R_{A, B}\right) \subset V\left(R_{A, B}, B(B(H))\right)$.

Let $E$ be a Banach space. We say that $S \in B(E)$ is an isometry if $\|S x\|=\|x\|$ for all $x \in E$. If $S$ is an invertible isometry, then its inverse $S^{-1}$ is also an isometry, and we have

$$
V\left(S T S^{-1}, B(E)\right)=V(T, B(E)), \quad T \in B(E) .
$$

In the case of a Hilbert space, an invertible isometry is unitary and its inverse is the adjoint.

Let $U$ and $V$ be two unitaries operators on $H$; then

$$
R_{U A U^{*}, V B V^{*}}=M_{U, V^{*}} R_{A, B} M_{U^{*}, V},
$$

with $M_{U, V^{*}}$ being an invertible isometry and its inverse being $M_{U^{*}, V}$.

From this result, we deduce that

$$
\begin{aligned}
V\left(R_{U A U^{*}, V B V^{*}}, B(B(H))\right) & =V\left(M_{U, V^{*}} R_{A, B} M_{U^{*}, V}, B(B(H))\right) \\
& =V\left(R_{A, B}, B(B(H))\right) .
\end{aligned}
$$

Now, using Lemma 3, we get

$$
\begin{gathered}
W\left(\sum_{i=1}^{k} U A_{i} U^{*} V B_{i} V^{*}\right) \subset V\left(R_{A, B}, B(B(H))\right), \\
\bigcup_{U, V \in U(H)} W\left(\sum_{i=1}^{k} U A_{i} U^{*} V B_{i} V^{*}\right) \subset V\left(R_{A, B}, B(B(H))\right) .
\end{gathered}
$$

But, the numerical range is closed and the product of two unitaries is also an unitary, hence:

$$
\begin{aligned}
& {\left[\bigcup_{U \in U(H)} W\left(\sum_{i=1}^{k} U A_{i} U^{*} B_{i}\right)^{-}\right]^{-}} \\
& \quad=\left[\bigcup_{V \in U(H)} W\left(\sum_{i=1}^{k} A_{i} V B_{i} V^{*}\right)^{-}\right]^{-} \subset V\left(R_{A, B}, B(B(H))\right) .
\end{aligned}
$$

So, we have proved the second inclusion of Theorem 1 . 
For the other inclusion, we will use the two following theorems.

Theorem 4 (See [11]). Let $\mathscr{A}$ be Banach algebra. For $a \in \mathscr{A}$, one has

$$
V(a, \mathscr{A})=\bigcap_{z \in \mathbf{C}}\{\lambda:|\lambda-z| \leq\|a-z\|\} .
$$

The norm of an elementary operator is defined by

$$
\left\|R_{A, B}\right\|=\operatorname{Sup}\left\{\left\|R_{A, B}(X)\right\|:\|X\| \leq 1\right\} .
$$

Let $\mathscr{A}$ be $C^{*}$-algebra. An element $U \in \mathscr{A}$ is said to be unitary if $U^{*} U=U U^{*}=I$. In the following, $U(\mathscr{A})$ denote the set of unitaries in $\mathscr{A}$.

Theorem 5 (Russo-Dye [12]). Let $\mathscr{A}$ be $C^{*}$-algebra. Let $A=$ $\left(a_{1}, \ldots, a_{k}\right)$ and $B=\left(b_{1}, \ldots, b_{k}\right)$ be two $k$-tuples of elements in $\mathscr{A}$. Then, one has

$$
\left\|R_{A, B}\right\|=\operatorname{Sup}\left\{\left\|R_{A, B}(U)\right\|: U \in U(\mathscr{A})\right\} .
$$

We return now to the proof of the main theorem.

Proof. We need only to show the inclusion " $\mathrm{C.}$ " By Theorem 4, we have

$$
V\left(R_{A, B}, B(B(H))\right)=\bigcap_{z \in \mathbf{C}}\left\{\lambda:|\lambda-z| \leq\left\|R_{A, B}-z\right\|\right\} .
$$

And, by Theorem 5, we get

$$
\left\|R_{A, B}-z\right\|=\operatorname{Sup}\left\{\left\|\sum_{i=1}^{k} A_{i} U B_{i}-z U\right\|: U \in U(H)\right\} .
$$

But $\|U T\|=\|T\|$ for all $T \in B(H)$ and $U \in U(H)$. Hence,

$$
\left\|R_{A, B}-z\right\|=\operatorname{Sup}\left\{\left\|\sum_{i=1}^{k} U^{*} A_{i} U B_{i}-z I_{H}\right\|: U \in U(H)\right\} .
$$

Hence, if $\lambda \in V\left(R_{A, B}, B(B(H))\right)$, then, for all $z \in \mathbf{C}$,

$$
\lambda \in\left\{|\mu-z| \leq\left\|R_{A, B}-z I_{B(H)}\right\|\right\} .
$$

Let $\epsilon>0$ fixed, there exists a unitary $U_{\epsilon}$ such that

$$
\left\|R_{A, B}-z I_{B(H)}\right\| \leq\left\|\sum_{i=1}^{k} U_{\epsilon}^{*} A_{i} U_{\epsilon} B_{i}-z I_{H}\right\|+\epsilon .
$$

Now, using Theorem 4, we have

$$
\begin{aligned}
& W\left(\sum_{i=1}^{k} U_{\epsilon}^{*} A_{i} U_{\epsilon} B_{i}\right)^{-} \\
& \quad=\bigcap_{z \in \mathbf{C}}\left\{\lambda:|\lambda-z| \leq\left\|\sum_{i=1}^{k} U_{\epsilon}^{*} A_{i} U_{\epsilon} B_{i}-z I_{H}\right\|\right\} .
\end{aligned}
$$

So, there exists $\mu \in W\left(\sum_{i=1}^{k} U_{\epsilon}^{*} A U_{\epsilon} B\right)$ such that $|\lambda-\mu| \leq \epsilon$. But $\epsilon$ is arbitrary, $\lambda \in\left[\cup_{U \in U(H)} W\left(\sum_{i=1}^{k} U^{*} A_{i} U B_{i}\right)^{-}\right]^{-}$. This finishes the proof of the main theorem.

\section{Some Applications}

It is well known that, for the spectrum, if $A, B \in B(H)$, then we have

$$
\sigma(A B) \cup\{0\}=\sigma(B A) \cup\{0\} .
$$

For the numerical range, this not true, but we can deduce the following corollary from the proof of Theorem 1 .

Corollary 6. For all $A, B \in B(H)$, one has

$$
\left[\bigcup_{U \in U(H)} W\left(A U B U^{*}\right)^{-}\right]^{-}=\left[\bigcup_{U \in U(H)} W\left(U B U^{*} A\right)^{-}\right]^{-} .
$$

The numerical radius of an operator $T \in B(E)$ is denoted by $v(T)$ and defined by

$$
v(T)=\sup \{|\lambda|: \lambda \in V(T, B(E))\} .
$$

Corollary 7. Let $A=\left(A_{1}, \ldots, A_{k}\right)$ and $B=\left(B_{1}, \ldots, B_{k}\right)$ be two $k$-tuples of elements in $B(H)$. Then, one has

$$
v\left(R_{A, B}\right)=\operatorname{Sup}\left\{v\left(\sum_{i=1}^{k} U^{*} A_{i} U B_{i}\right): U \in U(H)\right\} .
$$

In particular, for $A, B \in B(H)$,

$$
\begin{aligned}
v\left(\delta_{A, B}\right) & =\sup _{U \in U(H)} v\left(U A U^{*}-B\right) \\
& =\sup _{U \in U(H)} v\left(A-U B U^{*}\right) \\
& =\sup _{U, V \in U(H)} v\left(U A U^{*}-V B V^{*}\right) .
\end{aligned}
$$

Let $K$ be a nonempty subset of the plane and let

$$
\operatorname{diam}(K)=\sup _{\alpha, \beta \in K}|\alpha-\beta|
$$

From Corollary $7(B=A)$, one has

$$
\operatorname{diam}(W(A))=\sup \left\{v\left(U A U^{*}-A\right): U \in U(H)\right\} .
$$

So, the diameter of the numerical range $W(A)$ is equal to the diameter of the $v$-unitary orbit of the operator $A$.

\section{Conflict of Interests}

The author declares that there is no conflict of interests regarding the publication of this paper.

\section{References}

[1] F. F. Bonsall and J. Duncan, Numerical Ranges Vol I, Cambridge University Press, New York, NY, USA, 1973.

[2] F. F. Bonsall and J. Duncan, Numerical Rangesvol Vol II, Cambridge University Press, New York, NY, USA, 1973.

[3] K. E. Gustafson and D. K. M. Rao, Numerical Range: The Field of Values of Linear Operators and Matrices, Springer, New York, NY, USA, 1997. 
[4] C. K. Fong, Some Aspects of Derivations on B(H), University of Toronto, Seminar Notes, 1978.

[5] J. Kyle, "Numerical ranges of derivations," Proceedings of the Edinburgh Mathematical Society, vol. 21, no. 1, pp. 33-39, 1979.

[6] K. Mattila, "Complex strict and uniform convexity and hyponormal operators," Mathematical Proceedings of the Cambridge Philosophical Society, vol. 96, no. 3, pp. 483-493, 1984.

[7] S.-Y. Shaw, "On numerical ranges of generalized derivations and related properties," Australian Mathematical Society Journal A, vol. 36, no. 1, pp. 134-142, 1984.

[8] A. Seddik, "The numerical range of elementary operators. II," Linear Algebra and Its Applications, vol. 338, pp. 239-244, 2001.

[9] L. A. Fialkow, "Structural properties of elementary operators," in Elementary Operators and Applications (Blaubeuren, 1991), pp. 55-113, World Scientific, River Edge, NJ, USA, 1992.

[10] R. Schatten, Norm Ideals of Completely Continuous Operators, Springer, Berlin, Germany, 1960.

[11] J. G. Stampfli and J. P. Williams, "Growth conditions and the numerical range in a Banach algebra," The Tohoku Mathematical Journal, vol. 20, pp. 417-424, 1968.

[12] B. Russo and H. A. Dye, "A note on unitary operators in $C^{*}$ algebras," Duke Mathematical Journal, vol. 33, pp. 413-416, 1966. 


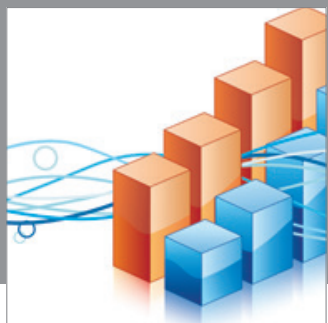

Advances in

Operations Research

mansans

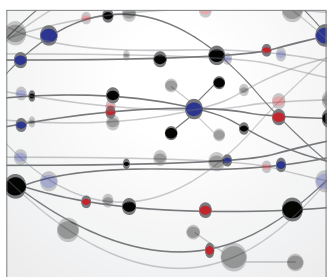

The Scientific World Journal
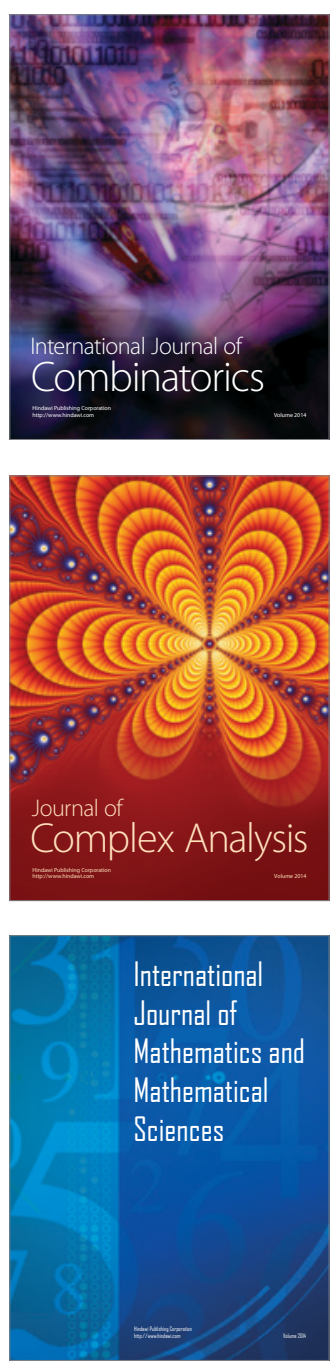
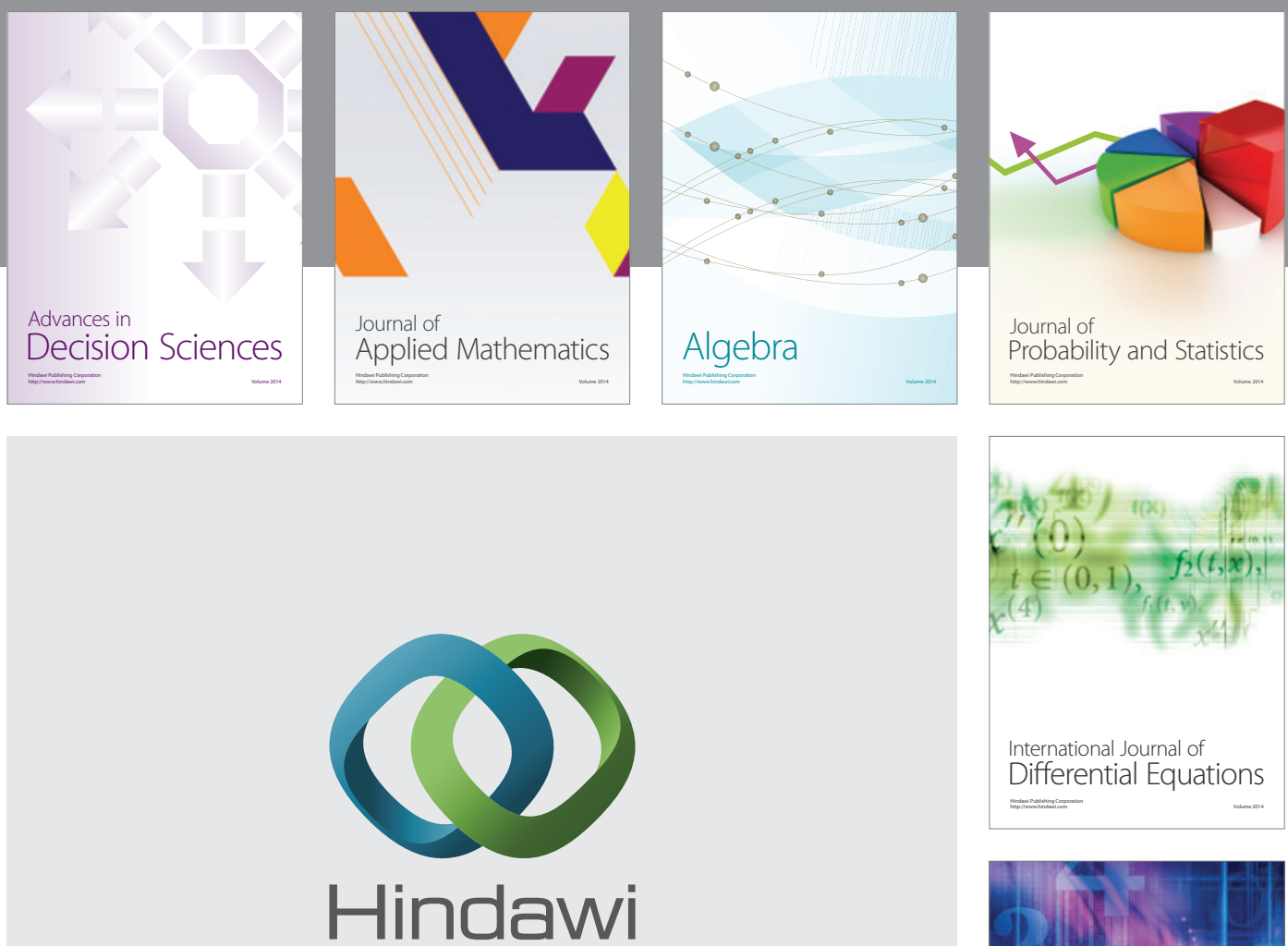

Submit your manuscripts at http://www.hindawi.com
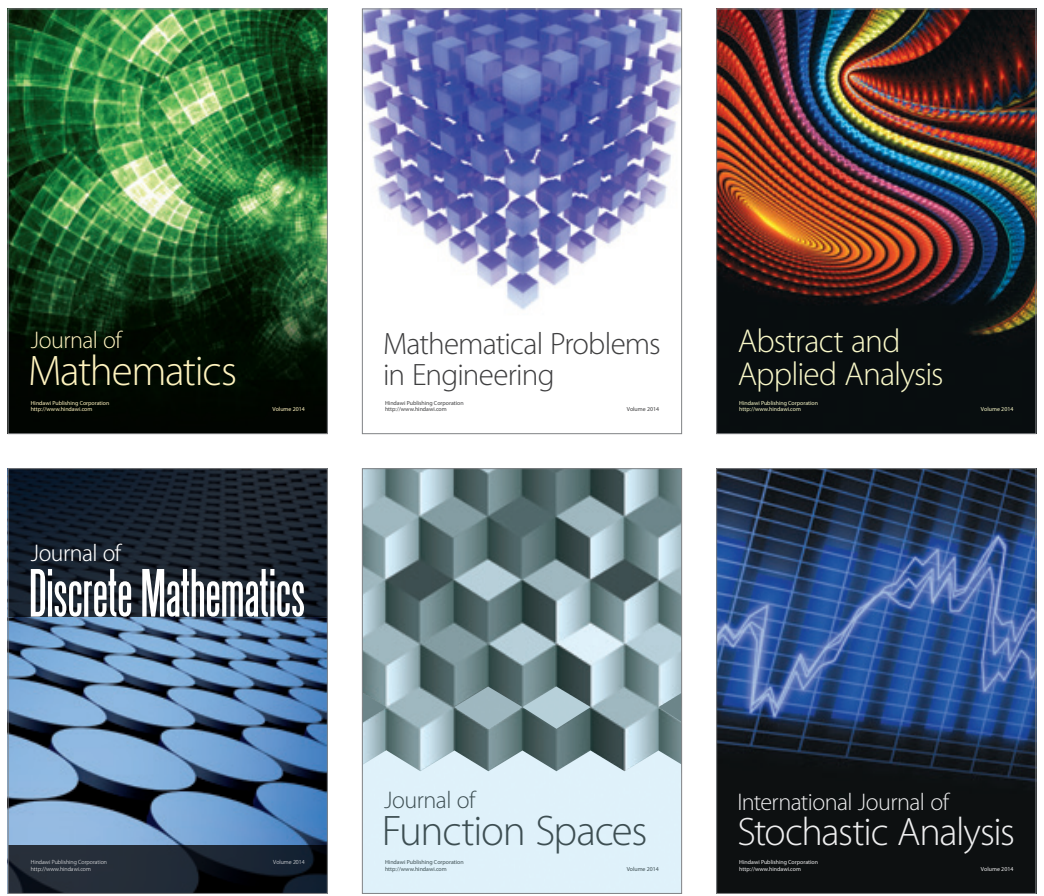

Journal of

Function Spaces

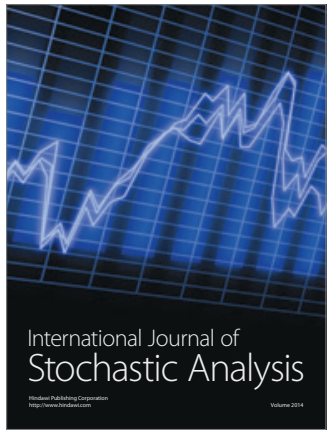

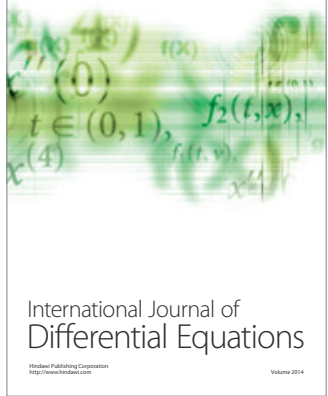
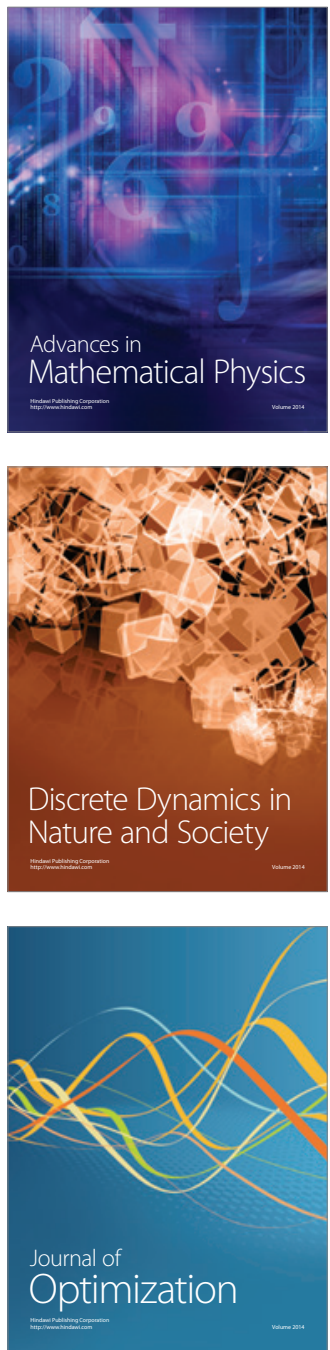